\title{
Phase-field simulation of anisotropic crack propagation in ferroelectric single crystals: effect of microstructure on the fracture process
}

\author{
Amir Abdollahi and Irene Arias \\ Laboratori de Càlcul Numèric (LaCàN), Departament de Matemàtica Aplicada III, \\ Universitat Politècnica de Catalunya (UPC), Campus Nord UPC-C2, E-08034 \\ Barcelona, Spain \\ E-mail: irene.arias@upc.edu
}

\begin{abstract}
Crack propagation during the indentation test of a ferroelectric single crystal is simulated using a phase-field model. This model is based on variational formulations of brittle crack propagation and domain evolution in ferroelectric materials. Due to the high compressive stresses near the indenter contact faces, a modified regularized formulation of the variational brittle fracture is coupled with the material model to prevent crack formation and interpenetration in the compressed regions. The simulation results show that the radial cracks perpendicular to the poling direction of the material propagate faster than the parallel ones, which is in agreement with experimental observations. This anisotropy in the crack propagation is due to interactions between the material microstructure and the radial cracks, as captured by the phase-field simulation.
\end{abstract}




\section{Introduction}

The unique electromechanical coupling properties of ferroelectrics make them ideal materials for use in micro-devices as sensors, actuators and transducers. Because of their brittleness, the design of the electromechanical devices requires the understanding of the complex fracture behavior in these materials. Complexity stems mainly from the nonlinear interactions of the mechanical and electrical fields in the vicinity of the crack, with localized switching phenomena, i.e. formation and evolution of ferroelectric domains [1-4]. Domain switching has been claimed as the main mechanism behind the fracture behavior of ferroelectric materials, in particular fracture toughness anisotropy.

The Vickers indentation technique is commonly used to investigate this anisotropy [

Experiments show that cracking along the poling direction of the material has a shorter length and consequently a higher effective fracture toughness than that normal to the poling direction.

Numerous investigations have been conducted to study the fracture of ferroelectrics

for excellent theoretical and experimental reviews). The linear theory of

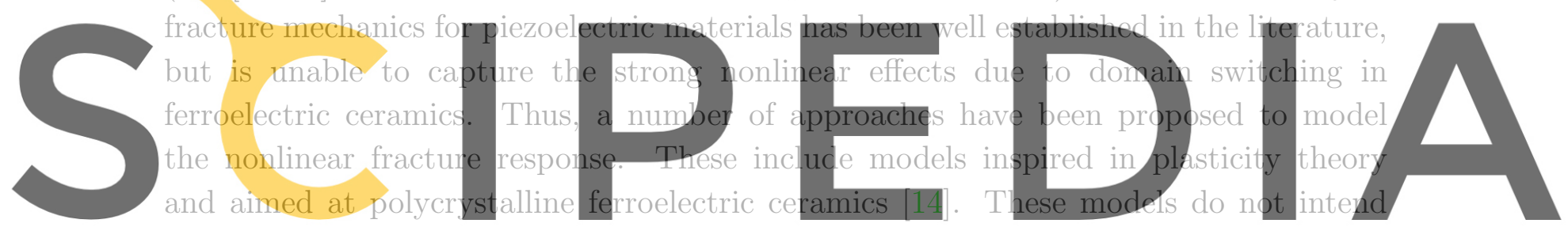

to describe explicitly the domain formation, but rather the effective phenomenology

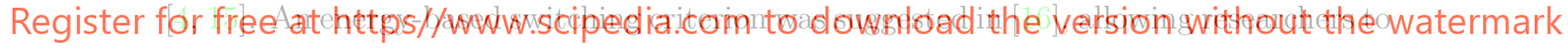
analyze the local phase transformations near the crack tip under the assumption of smallscale switching [17-19]. In the last years, phase-field or time-dependent DevonshireGinzburg-Landau (TDGL) models have gained a growing interest since they aim at explicitly describing the formation and evolution of individual ferroelectric domains in the framework of continuum mechanics [20-24]. See [25] and [26] for related models in micromagnetics. These microstructural models have specifically been applied to fracture, in all cases with a fixed crack. The nucleation and growth of domains near crack tips have been studied under applied electromechanical loadings, and the influence on the stress field and the mechanical and electromechanical $J$-integrals have been reported [27-29]. For completeness, we mention that cohesive theories aimed at fracture in ferroelectric materials have been proposed [30, 31].

The main objective of this paper is to introduce a model to explain the anisotropic crack growth under Vickers indentation loading, linking the microstructural details with the macroscopic observable response. We have recently proposed a phase-field model for the coupling of brittle crack propagation and microstructure evolution in ferroelectric 
single crystals [32]. The model couples a variational regularized model of brittle fracture [33-36] with a Devonshire-Ginzburg-Landau phase-field model for the microstructure evolution [20]. In this model, a system of partial differential equations governing the phase-fields accomplishes at once (1) the tracking of the interfaces in a smeared way (cracks, domain walls) and (2) the modeling of the interfacial phenomena (domain wall energies, crack face boundary conditions). This computational approach smears both the crack and the domain wall, and allows naturally for crack and domain nucleation, crack branching, crack and domain wall merging, and interactions between multiple cracks and domains. However this flexibility comes at the expense of a high computational cost, since the width of the phase-field regularizations of the domain wall and the crack must be resolved by the discretization. This model has been used to analyze the quasi-static crack propagation and the ferroelectric domain formation and evolution under combined electromechanical loading by tackling the full complexity of the phenomenon. However the model, as presented in [32], is unable to simulate the aforementioned anisotropic crack growth. Here we introduce a modification in the formulation of the phase-field

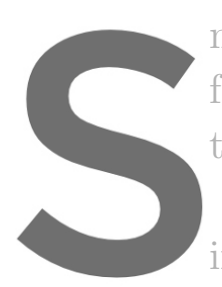
model endowing the for crack propagation a the total polarization

The model prop interpenetration condition in thon
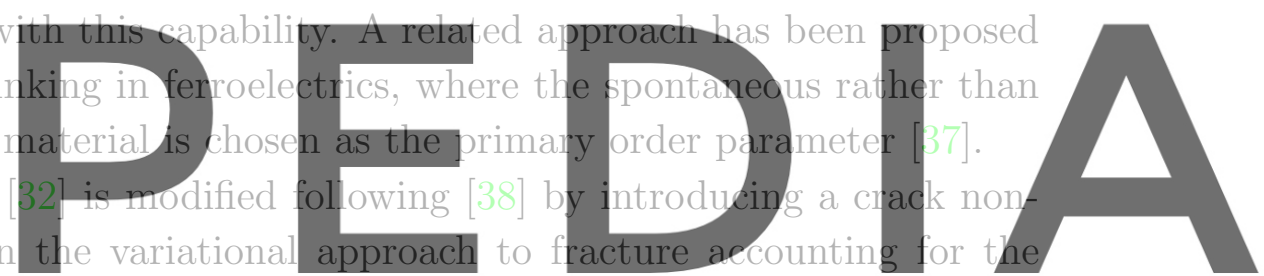

asymmetric behavior in tension and compression in the framework of linearized elasticity.

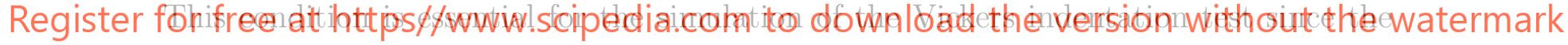

indentation loading induces high compressive stresses near the indenter contact faces.

Figure 1 presents the compression and tension zones around the indentation impression. Without the crack non-interpenetration condition, the variational approach would lead to crack propagation and interpenetration in the compression zones, along the indenter faces. Furthermore, radial crack propagation in tension zones is only obtained when this condition is considered in the model. For the ferroelectric response, we follow the Devonshire-Ginzburg-Landau phase-field model presented in [20] just as in [32].

The theory of the coupled phase-field model is described in Section 2. Simulation results indicate interactions between the material microstructure and the crack leading to the experimentally observed fracture anisotropy. These results are presented and discussed in Section 3. The last Section is the conclusion of this paper.

\section{Phase-field Model}

The proposed approach to brittle fracture in ferroelectric materials relies on the coupling of two energetic phase-field models, that of [20] for ferroelectric domain formation and 


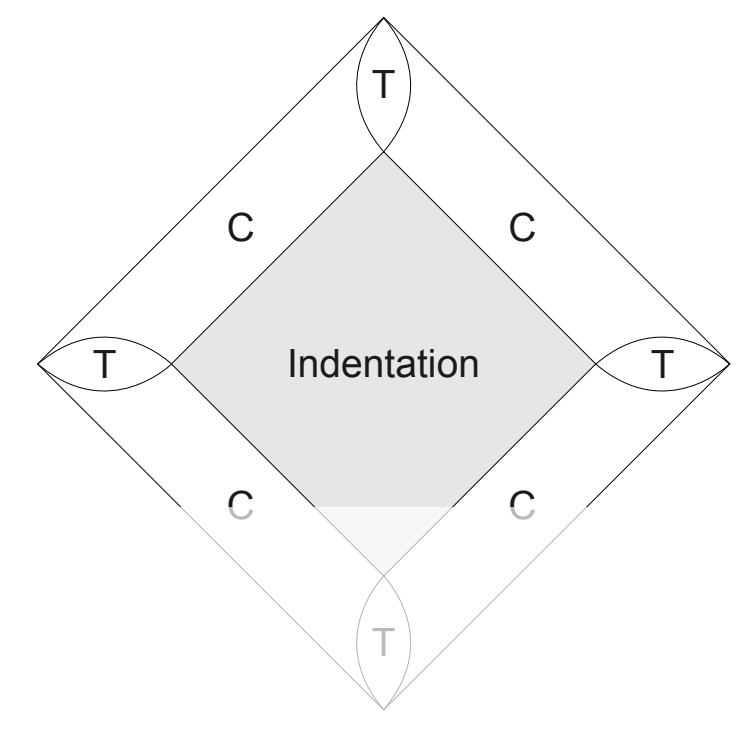

Figure 1. Compression $(\mathrm{C})$ and Tension (T) zones due to the indentation loading.

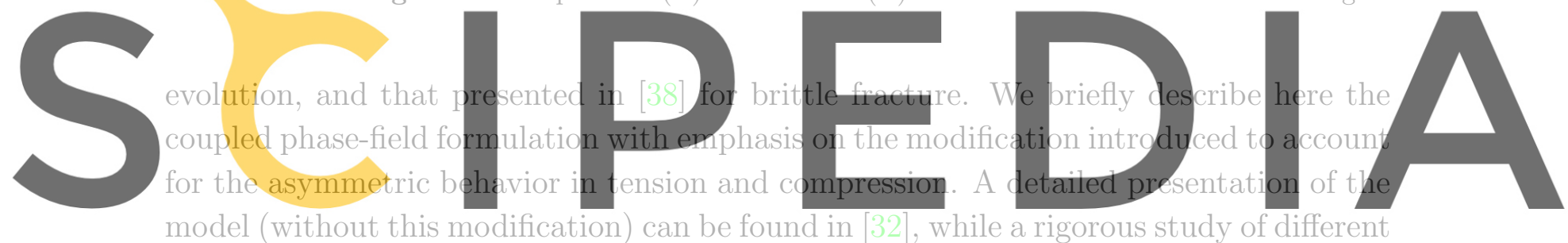

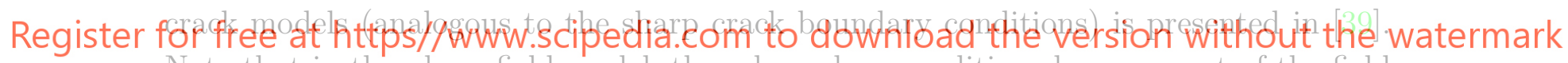

Note that in the phase-field model, these boundary conditions become part of the field

equations since the crack faces are represented by an internal layer.

\subsection{Electro-mechanical enthalpy}

We form a total electro-mechanical enthalpy of a possibly fractured ferroelectric material occupying a region $\Omega$ as

$$
\begin{aligned}
H[\boldsymbol{u}, \boldsymbol{p}, \phi, v]= & \int_{\Omega}\left[W_{e}(\varepsilon(\boldsymbol{u}), v)+W_{f}(\varepsilon(\boldsymbol{u}), \boldsymbol{p}, \phi, v)\right] \mathrm{d} \Omega \\
& +G_{c} \int_{\Omega}\left[\frac{(1-v)^{2}}{4 \kappa}+\kappa|\nabla v|^{2}\right] \mathrm{d} \Omega
\end{aligned}
$$

where body loads, volume charges, tractions and surface charges have been ignored for simplicity. In this functional, the bulk energy (first integral) competes with the surface energy (second integral). The constant $G_{c}$ is the critical energy release rate or the surface energy density in Griffith's theory [40]. The scalar field $v$ is the phase-field parameter describing a smooth transition in space between unbroken $(v=1)$ and broken $(v=0)$ 
states of the material. When the positive regularization parameter $\kappa$ tends to zero, this transition becomes sharper. The first integral in Eq. (1) is referred to as total bulk energy, $W_{e}$ being the part of the bulk energy density associated with the strain $\varepsilon$ and $W_{f}$ being the electro-mechanical energy density associated with the ferroelectric response. To account for the quite different fracture behavior in tension and compression, the energy density $W_{e}$ is written in [38] as

$$
W_{e}(\varepsilon, v)=\kappa_{0} \frac{\operatorname{tr}^{-}(\varepsilon)^{2}}{2}+\left(v^{2}+\eta_{\kappa}\right)\left(\kappa_{0} \frac{\operatorname{tr}^{+}(\varepsilon)^{2}}{2}+\mu \varepsilon_{D} \cdot \varepsilon_{D}\right)
$$

where $\kappa_{0}$ and $\mu$ are the bulk and shear modulus of the material, respectively. The decomposition of the trace of the strain tensor $\varepsilon$ in positive and negative parts are $\operatorname{tr}^{+}=\max (\operatorname{tr}(\varepsilon), 0)$ and $\operatorname{tr}^{-}=\max (-\operatorname{tr}(\varepsilon), 0)$ and $\varepsilon_{D}$ are the deviatoric components of the strain tensor. This decomposition is introduced to distinguish the contributions to the strain energy due to compression, expansion, and shear. In contrast to [32], here only the expansion and shear terms are multiplied by the jump set function $\left(v^{2}+\eta_{\kappa}\right)$

to prevent crack nucleation, propagation and interpenetration in compressed regions.

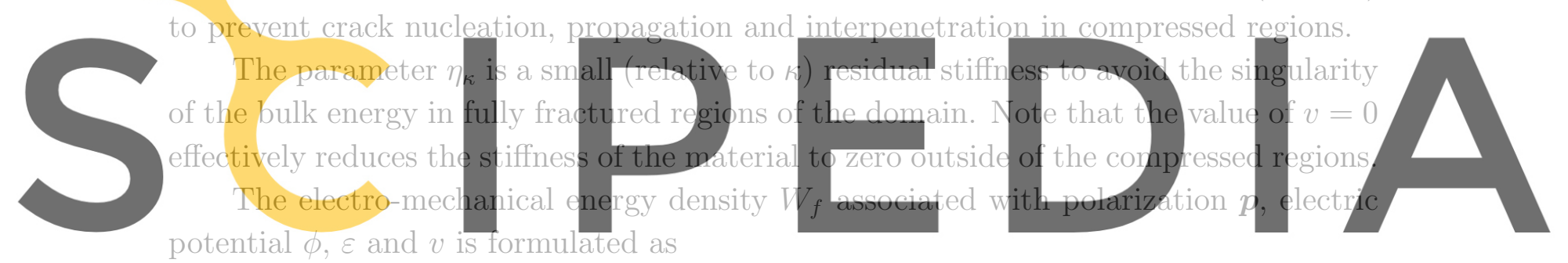

Register for free at https//www.scipedia.com to download the version without the watermark

$W_{f}(\varepsilon, p, \phi, v)=\left(v^{2}+\eta_{\kappa}\right)[U(\nabla p)+W(p, \varepsilon)]+\chi(p)-\frac{\varepsilon_{0}}{2}|\nabla \phi|^{2}+\nabla \phi \cdot p$,

where $U$ is the domain wall energy density penalizing sharp variations in the polarization, $\chi$ is the phase separation potential, $W$ is the electro-mechanical coupling energy density and $\varepsilon_{0}$ is the vacuum permittivity. The energy densities $\chi$ and $W$ penalize deviations from the spontaneous polarizations and strains of the material, hence introducing the anisotropy and nonlinearity of ferroelectric materials.

This particular formulation of the phase-field model encodes the asymmetric fracture response in tension and compression, as well as the assumed crack conditions. We assume the crack to be traction-free (i.e. $\boldsymbol{\sigma} \cdot \boldsymbol{n}=\mathbf{0}$ on the crack faces, $\boldsymbol{\sigma}$ and $\boldsymbol{n}$ being the stress tensor and the unit outward normal, respectively) and electrically permeable, which has been checked to be a reasonable approximation when the electrical discharge strength of the medium within the crack is small, in the order of that of air [41]. The permeable crack conditions assume that crack faces are closed and the electric field is not perturbed by the presence of the crack. Mathematically, these conditions lead 
to the continuity of the electric potential $\phi$ and the normal component of the electric displacement $\boldsymbol{D}$ across the crack faces, i.e.

$$
\phi^{+}=\phi^{-} \quad \text { and } \quad \boldsymbol{D}^{+} \cdot \boldsymbol{n}^{+}=\boldsymbol{D}^{-} \cdot \boldsymbol{n}^{-},
$$

where the superscripts + and - denote the top and bottom crack faces. In addition, free-polarization boundary conditions [42] are commonly assumed, implying that the gradients normal to the crack faces of the polarization components vanish at the crack faces, i.e.

$$
\frac{d p_{i}^{+}}{d n}=\frac{d p_{i}^{-}}{d n}=0,(i=1,2)
$$

It can be seen that, in the limit of vanishingly small regularization parameter, the proposed phase-field model recovers these conditions on the crack faces, as expected in the sharp crack model [39]. For a finite but small value of the regularization parameter $\kappa$, as used in practical computations, the resulting solutions satisfy the conditions in the vicinity of the smeared crack in an approximate but accurate way. Other electromechanical crack conditions can also be modeled similarly [39].
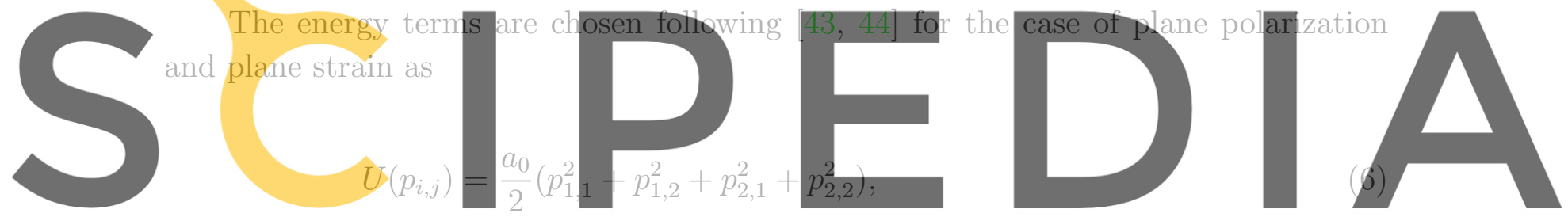

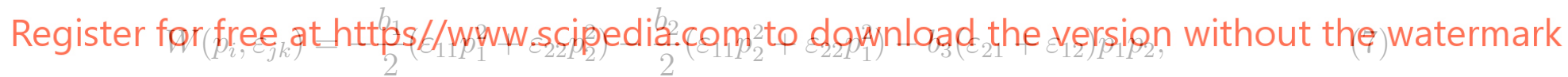

$\chi\left(p_{i}\right)=\frac{a_{1}}{2}\left(p_{1}^{2}+p_{2}^{2}\right)+\frac{a_{2}}{4}\left(p_{1}^{4}+p_{2}^{4}\right)+\frac{a_{3}}{2}\left(p_{1}^{2} p_{2}^{2}\right)+\frac{a_{4}}{6}\left(p_{1}^{6}+p_{2}^{6}\right)+\frac{a_{5}}{4}\left(p_{1}^{4} p_{2}^{4}\right)$,

where $a_{0}$ is the scaling parameter of the domain wall energy, $a_{i}(i=1, . ., 5)$ are the constants of the phase separation energy and $b_{i}(i=1,2,3)$ are the constants of the electro-machanical coupling terms. The phase separation potential $\chi$ includes only an eight-order term as proposed in [20]. This potential can be improved by adding other high order terms to reproduce the dielectric behavior of barium titanate single crystals in a more accurate way $[45,46]$. Nevertheless, note that the role of the eighth order cross-term $p_{1}^{4} p_{2}^{4}$ is the most important among all high order terms for the phase-field modeling of ferroelectric domains in the tetragonal phase. In fact, this term provides a reasonable energy barrier for $90^{\circ}$ domain switching, while allowing the other terms to fit the dielectric behavior of the material [20]. The combination of the energy functions $\chi$, $W$ and $W_{e}$ is the total Landau-Devonshire energy density furnishing a multi-well energy landscape with four minima corresponding to the four variants of the tetragonal phase. 


\subsection{Evolution}

The main objective of the coupled model is to capture interactions between the microstructure formation and evolution, and the crack propagation. For this reason, cracks should not propagate much faster than the microstructure relaxation. In the absence of detailed experimental or fundamental information on the relative kinetics of microstructure evolution and crack propagation, the field $v$ and the polarization $\boldsymbol{p}$ are selected as primary order parameters. The time evolution of the system results from the gradient flows of the total electro-mechanical enthalpy with respect to the primary variables, assuming that the displacement and the electric field adjust immediately to mechanical and electrostatic equilibrium (with infinite mobility), i.e.

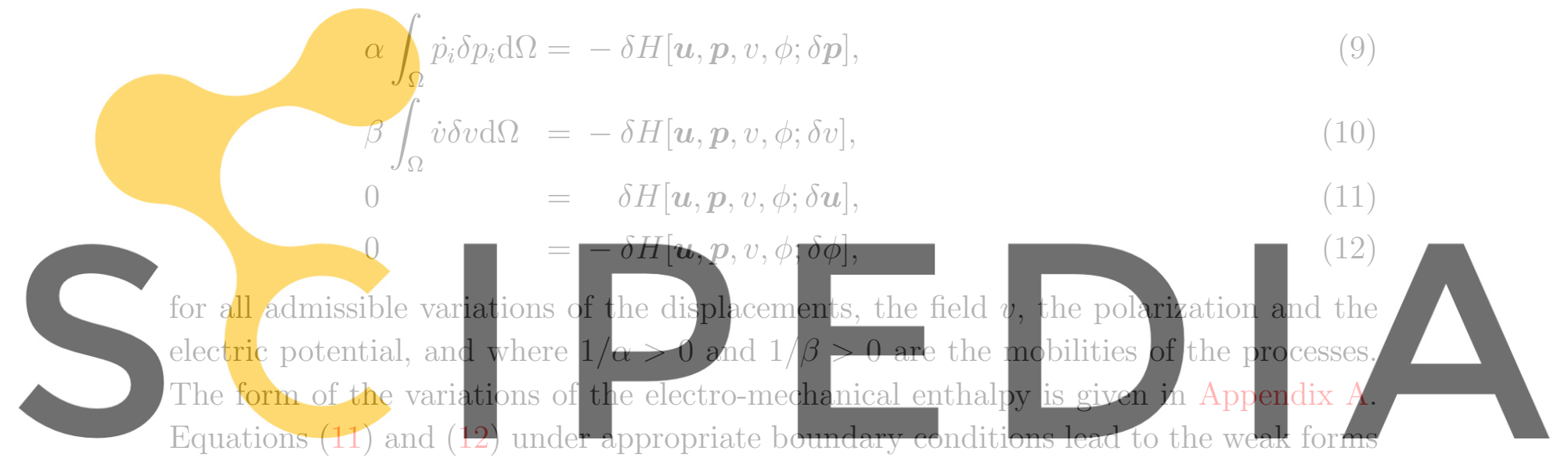

of the mechanical and electrostatic equilibria, respectively.

Register for frefe at https/www scipedia.fom to download the version without the watermark

a function of six degrees of freedom $u, p, \phi$ and $v$ but for a linear elastic body, it is convex and quadratic in $\phi$ and $v$ separately and it is convex and piecewise quadratic in $\boldsymbol{u}$. A multi-well energy landscape is also obtained with respect to $\boldsymbol{p}$ where each well corresponds to one of the four variants of the tetragonal phases. The minimization in Eq. (11) is non-smooth, and a quasi-Newton algorithm is applied to solve the mechanical equilibrium problem following [38]. These equations are discretized in time with a semiimplicit scheme.

The solution algorithm follows a straightforward staggered procedure. It is aimed at achieving steady states for both ferroelectric domains and brittle fracture in each load step. In what follows, superscripts refer to load steps and subscripts to iterations within a load step. For each load step there is an internal loop to reach reasonable tolerances for the $v$ field and the polarization $\boldsymbol{p}$, where $\delta_{v \text { field }}$ and $\delta_{\text {ferro }}$ are small tolerances respectively. The functions $\boldsymbol{g}(w)$ and $h(w)$ encode mechanical displacement and electric potential as a function of the load step on the Dirichlet boundary of the model. Since the crack should not be allowed to heal (irreversibility condition), when 
the value of $v$ decreases beyond a threshold $\gamma$, it is forced to zero and kept so for the rest of the calculation. After reaching a steady state for both the polarization and $v$ field, the values of $v^{n}, \boldsymbol{u}^{n}, \boldsymbol{p}^{n}$ and $\phi^{n}$ are recorded and the algorithm is repeated for the next load step.

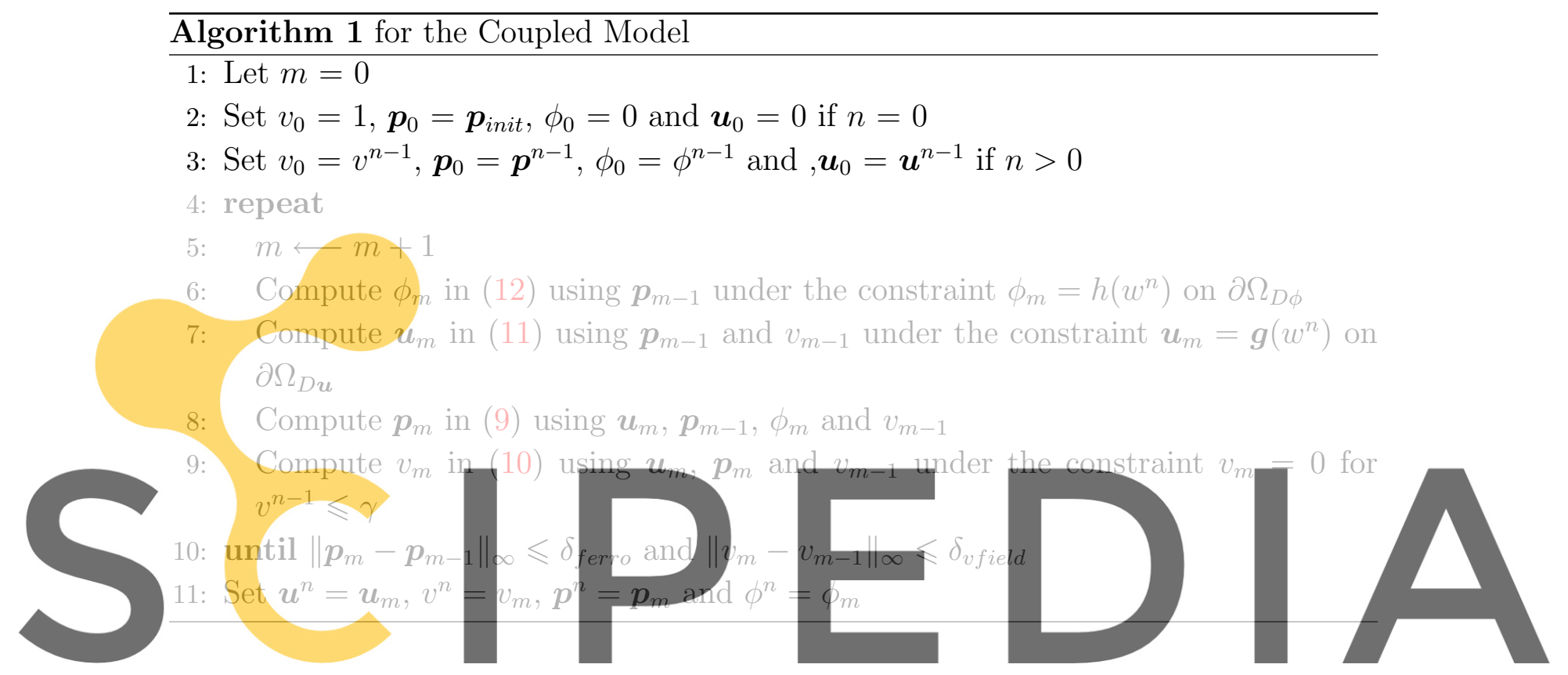

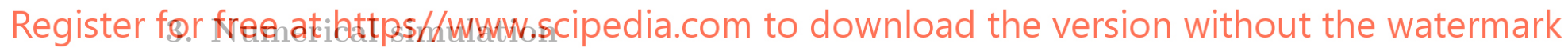

3.1. Computational domain and parameter setting

We consider an indentation impression lying inside a ferroelectric single crystal in two dimensions, as shown in Fig. 2. The indentation is included in the model by considering a square inner boundary. A monotonically increasing mechanical load is applied by pulling the indentation faces with uniform displacement such that $\boldsymbol{u}(w)=w \boldsymbol{n}$, $w$ being the load level in the quasi-static loading process. This type of mechanical loading is applied to the specimen by the indenter during a small growth of the indentation. It is assumed that the indenter faces are connected to the ground, therefore the electric potential is fixed to $\phi=0$ on the indentation faces. The computational domain represents a piece of a larger ferroelectric crystal, therefore mechanical displacement, electric potential and polarization should be uniformly distributed across the four outer edges. The following conditions are imposed on these boundaries: (1) $\boldsymbol{\sigma} \cdot \boldsymbol{n}=\mathbf{0},(2) \nabla \phi \cdot \boldsymbol{n}=0$ and (3) freepolarization, see Eq. (5). The initial polarization $\boldsymbol{p}_{0}=(0,1)$ is assigned to the model along the positive vertical direction, see Fig. 2. The normalized dimension of the domain 


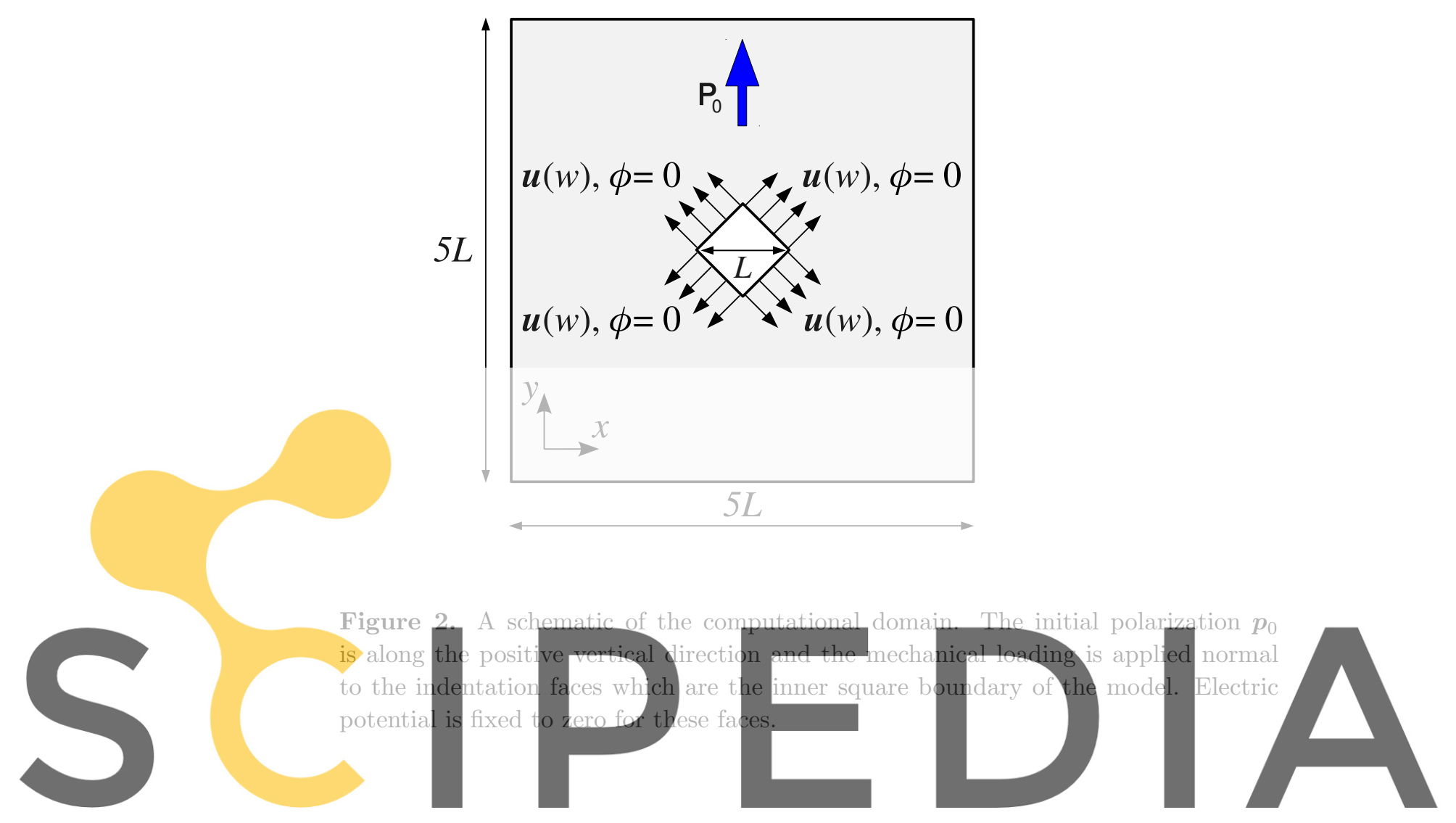

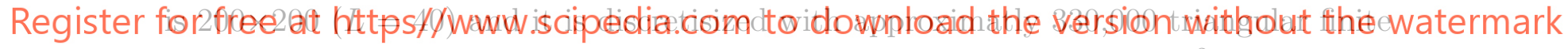

elements of different sizes. The fine mesh with element size $h \simeq 5 \times 10^{-2}$ is generated

in rectangular areas around the radial directions, where fracture is expected, while the rest of the domain is meshed with larger elements (of size $h \simeq 1$ ). A sample mesh is presented in Fig. 3.

For convenience, dimensionless variables are selected through the following normalizations: $x_{i}^{\prime}=x_{i} \sqrt{c_{0} / a_{0}} / p_{0}, p_{i}^{\prime}=p_{i} / p_{0}, t^{\prime}=t c_{0} / \mu p_{0}^{2}, \varepsilon_{0}^{\prime}=\varepsilon_{0} c_{0} / p_{0}^{2}, \phi^{\prime}=\phi / \sqrt{a_{0} c_{0}}$, $a_{1}^{\prime}=a_{1} p_{0}^{2} / c_{0}, a_{2}^{\prime}=a_{2} p_{0}^{4} / c_{0}, a_{3}^{\prime}=a_{3} p_{0}^{4} / c_{0}, a_{4}^{\prime}=a_{4} p_{0}^{6} / c_{0}, a_{5}^{\prime}=a_{5} p_{0}^{8} / c_{0}, b_{i}^{\prime}=b_{i} p_{0}^{2} / c_{0}$, $\mu^{\prime}=\mu / c_{0}$ and $\kappa_{0}^{\prime}=\kappa_{0} / c_{0}$, where $i=1,2,3$. The equations with normalized variables are the same as the original ones. The constants are chosen to fit the behavior of single crystals of barium titanate $\left(\mathrm{BaTiO}_{3}\right)$, taking $c_{0}=1 \mathrm{GPa}$ and spontaneous polarization $p_{0}=0.26 \mathrm{C} / m^{2}[20]$. The domain wall scaling parameter is set to $a_{0}=2.4 \times 10^{-10} \mathrm{Vm}^{3} / \mathrm{C}$. The normalized scaling parameter of the domain wall energy $a_{0}^{\prime}$ is used to adjust the domain wall width in the computational domain. This parameter has to be chosen such that the variation of the polarization can be resolved by the discretization while domain walls remain sufficiently sharp relative to the other dimensions of the problem. 


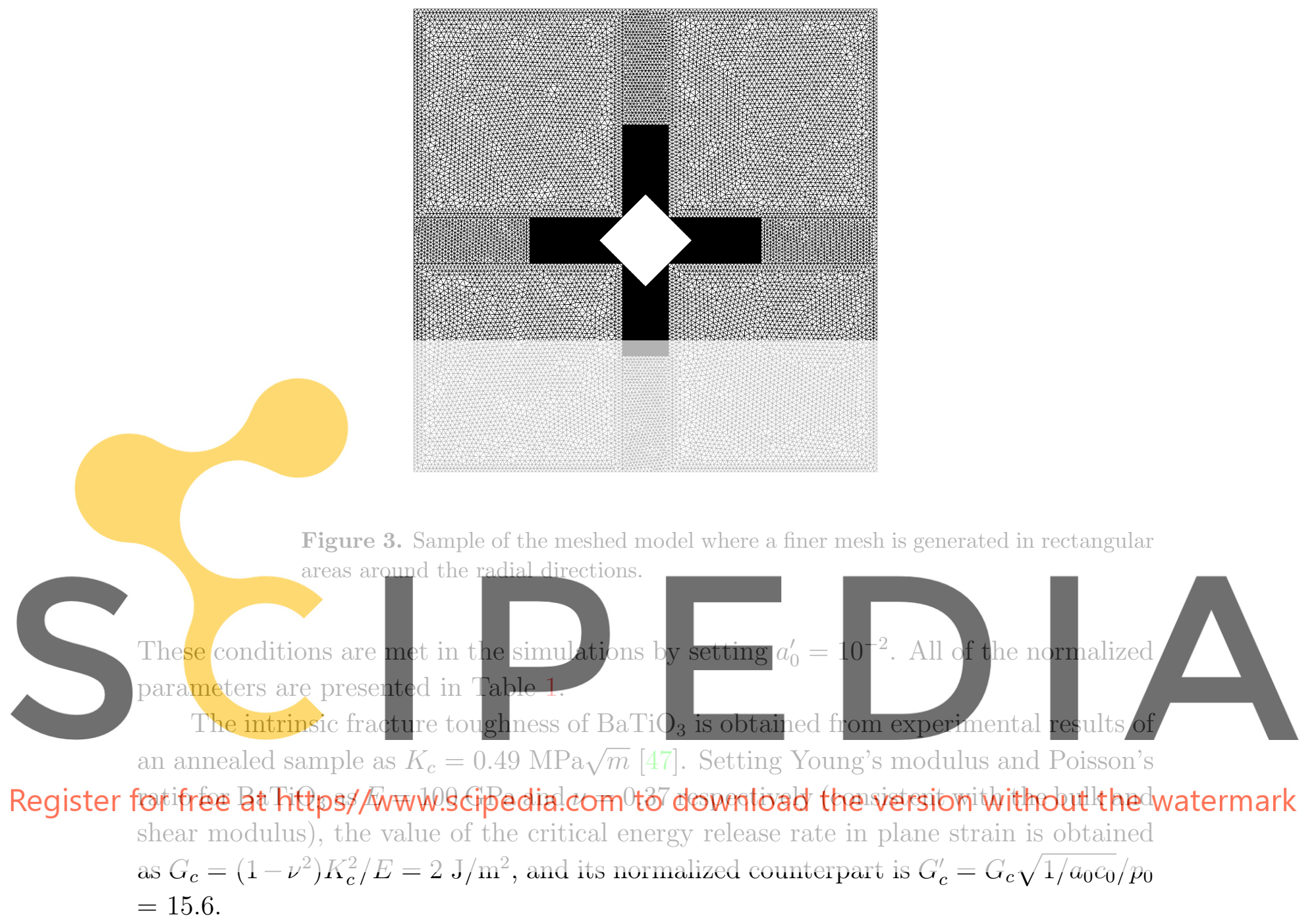

The value of the fracture regularization parameter $\kappa$ is chosen based on parametric studies of the discretized surface energy [36, 38]. For an accurate discretized surface energy, the element size $h$ should be much smaller than the regularization parameter $\kappa$. In addition, an accurate approximation of the sharp-crack model of brittle fracture calls for a sufficiently small value of $\kappa$ relative to the other dimensions of the problem. This in turn leads to extremely fine meshes with very high computational cost (the coupled model has six degrees of freedom per node in two dimensions). Numerical experiments indicate that setting $\kappa \sim h$ gives reasonable results, although the computed surface energy can be expected to be slightly overestimated [36, 38]. For the simulations of this paper, the regularization parameter is set to twice the finest element size as $\kappa=0.1$, i.e. $h / \kappa=0.5$.

The residual stiffness $\eta_{\kappa}$ must be chosen as small as possible to avoid adding too 
much artificial stiffness and permittivity to the elements in the fracture zone, while preserving the non-singularity of the stiffness matrices used for the solution of finite element equations. We take $\eta_{\kappa}=10^{-4}$ without any observed numerical instabilities in the simulations.

The remaining constants are chosen as follows: two tolerances $\delta_{\text {ferro }}=10^{-3}$ and $\delta_{\text {field }}=5 \times 10^{-3}$, the threshold to detect the fracture zone $\gamma=2 \times 10^{-2}$ and the inverse of the mobilities $\alpha=\beta=5 \times 10^{-2}$. Fifty load steps are computed $(n=50)$ with load increments of $\Delta w^{n}=5 \times 10^{-2}$. The normalized time step $\Delta t_{m}^{\prime}=10^{-2}$ leads to convergent and accurate solutions for the time integration of gradient flow equations in (9) and (10). The simulations are carried out on parallel processors using the finite element library of the Kratos multi-physics package [48].

Table 1. Normalized parameters

\begin{tabular}{ccccccc}
\hline$\kappa_{0}^{\prime}$ & $\mu^{\prime}$ & $b_{1}^{\prime}$ & $b_{2}^{\prime}$ & $b_{3}^{\prime}$ & $a_{0}^{\prime}$ \\
\cline { 1 - 7 } 146 & 36 & 1.4282 & -0.185 & 0.8066 & 0.01 \\
\hline$a_{1}^{\prime}$ & $a_{2}^{\prime}$ & $a_{3}^{\prime}$ & $a_{4}^{\prime}$ & $a_{5}^{\prime}$ & $\varepsilon_{0}^{\prime}$ & $G_{c}^{\prime}$ \\
\hline-0.007 & -0.009 & 0.018 & 0.0261 & 5 & 0.131 & 15.6 \\
\hline
\end{tabular}

\subsection{Numerical results and discussion}

Two snapshots of the crack propagation are presented in Fig. 4. The value of $v$ starts to decrease towards zero around the vertices of the indentation as the load step increases. After reaching the zero value at load step $w=0.5$, i.e. crack initiation, the fracture zone grows along the four radial directions as shown at two sample load steps $w=1.5$ and $w=2.5$ in Fig. 4(a) and Fig. 4(b) respectively.

To evaluate the radial crack growth, four equally large areas around the corners of the indentation are considered in Fig. 4(b) and the value of surface energy (the second integral in (1)) is obtained for each zone. Note that the surface energy is an indirect measure of the crack length. The surface energy graphs are shown in Fig. 5. It is obvious in this figure that surface energies of zones 1 and 3 follow nearly the same path. This also holds for zones 2 and 4. Interestingly, the surface energies of zones 1 and 3 are larger than those of zones 2 and 4, i.e. the perpendicular cracks to the polarization are longer than the parallel ones. This is a clear evidence of the anisotropic crack propagation in agreement with experimental observations [5-10]. For comparison purposes, we also compute the surface energy for an elastic single-phase material by fixing the initial polarization to zero $\left(\boldsymbol{p}_{0}=\mathbf{0}\right)$. For this simulation, there is no need 


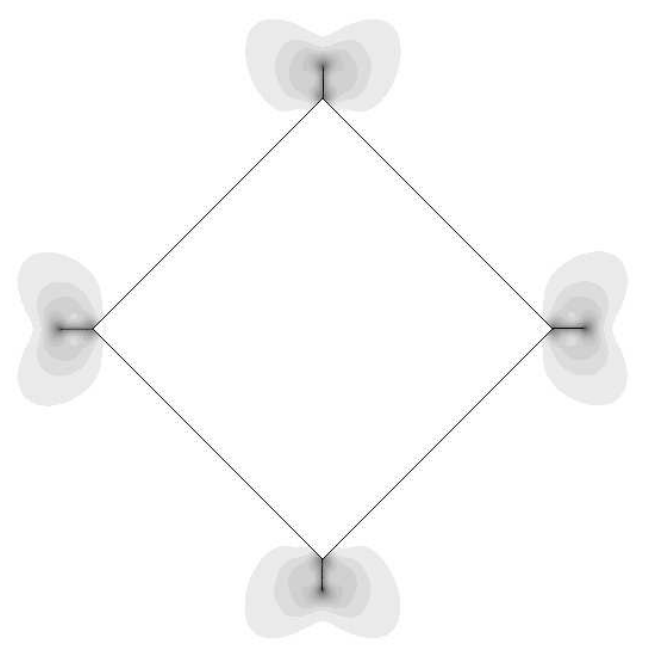

(a)

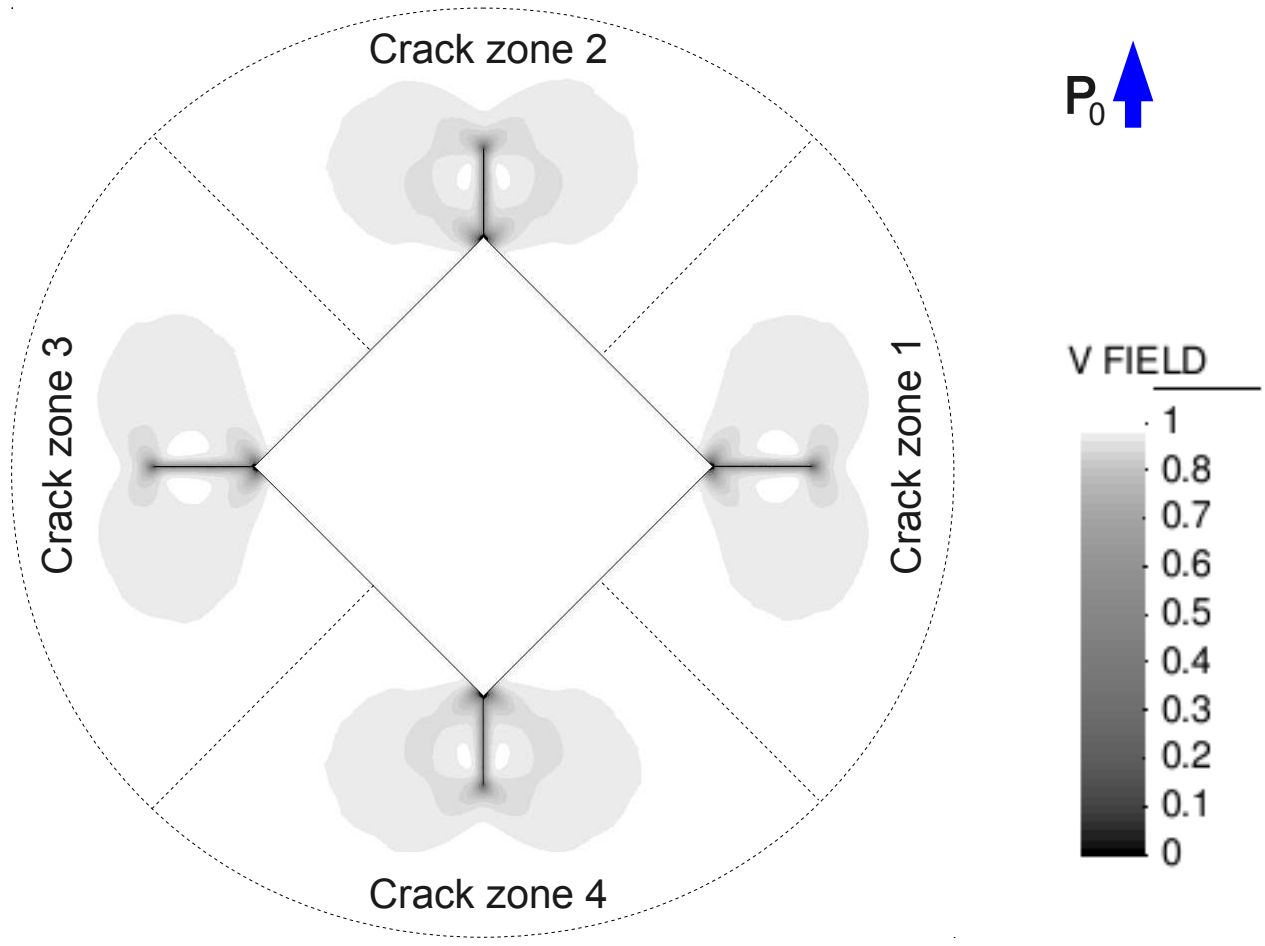

(b)

Figure 4. Two snapshots of the fracture evolution: contour plots of the field $v$ at load steps (a) $w=1.5$ (b) $w=2.5$. Four equally large areas around the corners of the indentation are considered to obtain the surface energy evolution of the four radial cracks (crack zones $1-4$ ) shown in Fig. 5. 


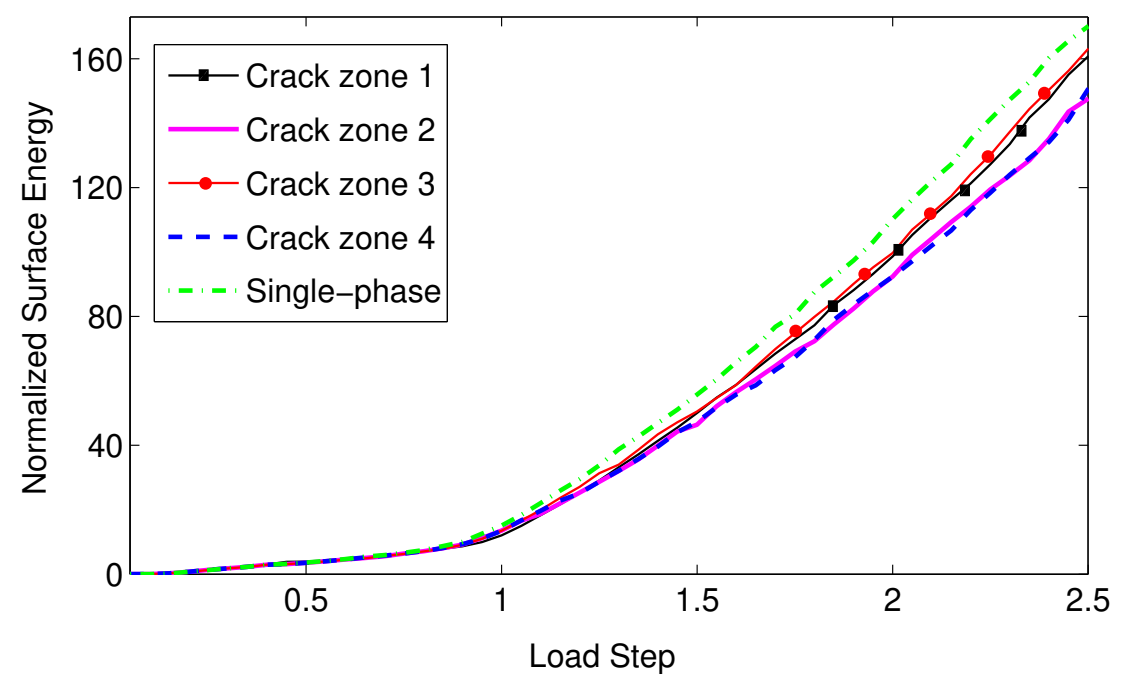

Figure 5. Evolution of the normalized surface energy of the four zones (crack zones $1-4)$ marked in Fig. 4(b) as a function of the load step $w$. The single-phase graph is obtained for one of these zones considering the elastic material with zero fixed polarization.

to solve for the polarization and the electric potential, and the unknowns reduce to $\boldsymbol{u}$ and $v$ (skip lines 6 and 8 in Algorithm 1). The energy graphs of all the four zones for the single-phase material fall on top of each other, a testament of the isotropic crack propagation. One of these graphs is presented in Fig. 5 (marked as single-phase graph).

The origin of the observed fracture toughness anisotropy can be found in the domain switching during crack growth. Figure 6 presents two snapshots of the domain evolution in an area around the indentation at load steps $w=1.2$ and $w=1.8$. Ferroelastic domain switching occurs around the tip of the parallel cracks (cracks 2 and 4), where the horizontal components of the polarization vectors indicate wing-shaped domains or twins. This kind of switching is induced by the high tensile stresses near the crack tip tending to elongate the material in the horizontal direction in front of the parallel cracks. The elongation near the perpendicular cracks (cracks 1 and 3 ) in the vertical direction is also observed without any ferroelastic domain switching since the polarization is aligned with the tensile stresses. Due to the absence of ferroelastic domain switching, the perpendicular cracks grow more than parallel ones and the effective fracture toughness is lower perpendicular to the poling direction. Indeed, part of the high concentration of stored elastic energy near the parallel crack tips can be released by aligning the longer axis of the crystal with the tensile stresses through switching rather than by propagating 
the crack. Figures 6(a) and 6(b) show that the wing-shaped switched domains grow gradually with the monotonically increasing load and the toughening effect becomes progressively more pronounced for the parallel cracks, as shown in Fig. 5. The 90 domain switching-induced toughening is also reported in other experiments of crack propagation in $\mathrm{BaTiO}_{3}[47,49,50]$. It is also obvious in Fig. 5 that the elastic singlephase material has the lowest fracture toughness due to the absence of the microstructure effect. The material elongation in the vertical direction in front of the perpendicular cracks leads to a toughening enhancement with respect to the single-phase material (compare the single-phase graph and the graphs of zones 1 and 3 in Fig. 5).

\section{Conclusions}

We perform, to the best of our knowledge, the first simulation of Vickers indentation crack growth in ferroelectric single crystals with the goal of evaluating the effect of the microstructure on the fracture process. This is done by formulating a coupled phasefield model based on variational formulations of brittle crack propagation and domain evolution in ferroelectric materials. The simulation results show that radial cracks perpendicular to the poling direction of the material propagate faster than parallel ones, which is in agreement with experimental observations. This anisotropy in the fracture toughness follows from the fully coupled interactions between the material microstructure and the crack propagation. $90^{\circ}$ ferroelastic switching induced by the intense crack-tip stress field is observed near the parallel cracks, which is believed as the main fracture toughening mechanism in ferroelectric materials. These results prove the potential of our coupled phase-field model to elucidate the fracture behavior of ferroelectric ceramics, whose technological implications are very important.

We also suggest that more work is needed to produce predictive simulations of such complex phenomena. In particular, a more precise evaluation of the microstructure effect will be obtained by extending the proposed model to three dimensions and running longer simulations producing more extended cracks. Also, it is widely accepted that the crack face electro-mechanical boundary conditions strongly affect the crack propagation in piezoelectric and ferroelectric ceramics, and thus more physically realistic crack conditions should be analyzed. Another important issue is the quantification and relative magnitude of the mobility parameters $\alpha$ and $\beta$, which can have an important effect on the resulting response. Finally, although domain switching is claimed to be the main source of fracture toughness anisotropy, the intrinsic anisotropy of the crystal, i.e. the different surface energy in different cleavage planes, is bound to have an effect as well. These topics are currently under investigation. 

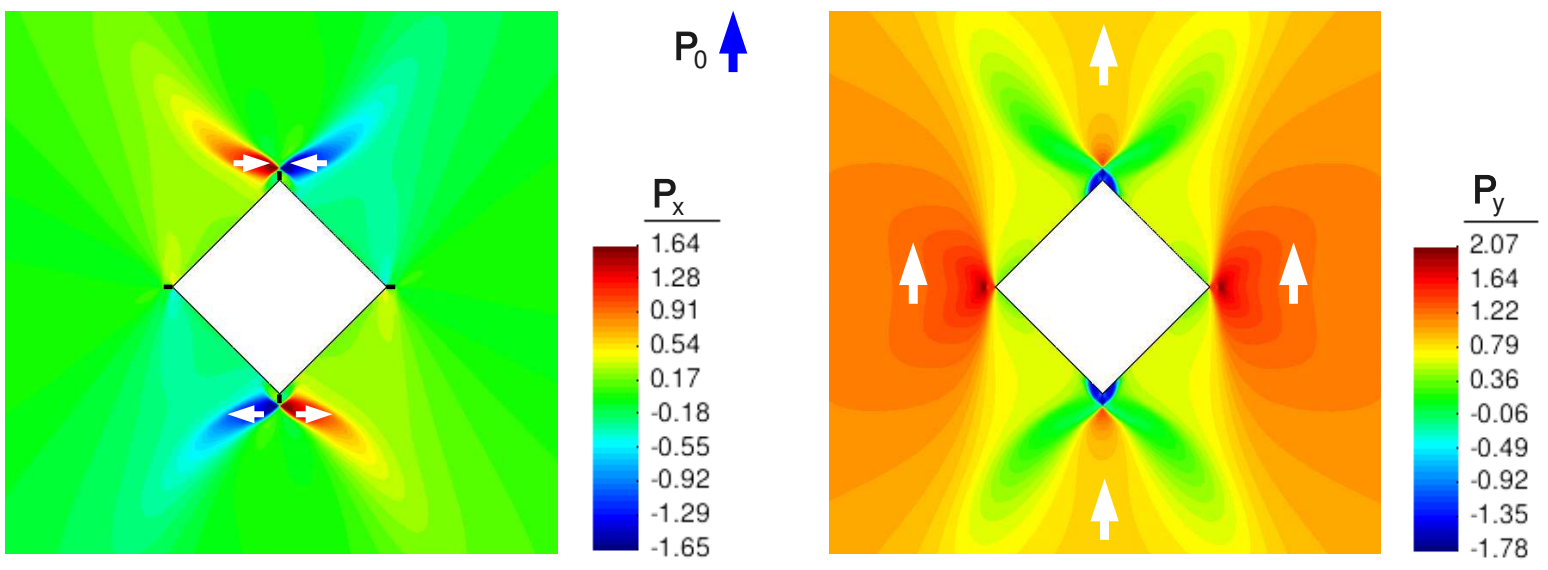

(a)
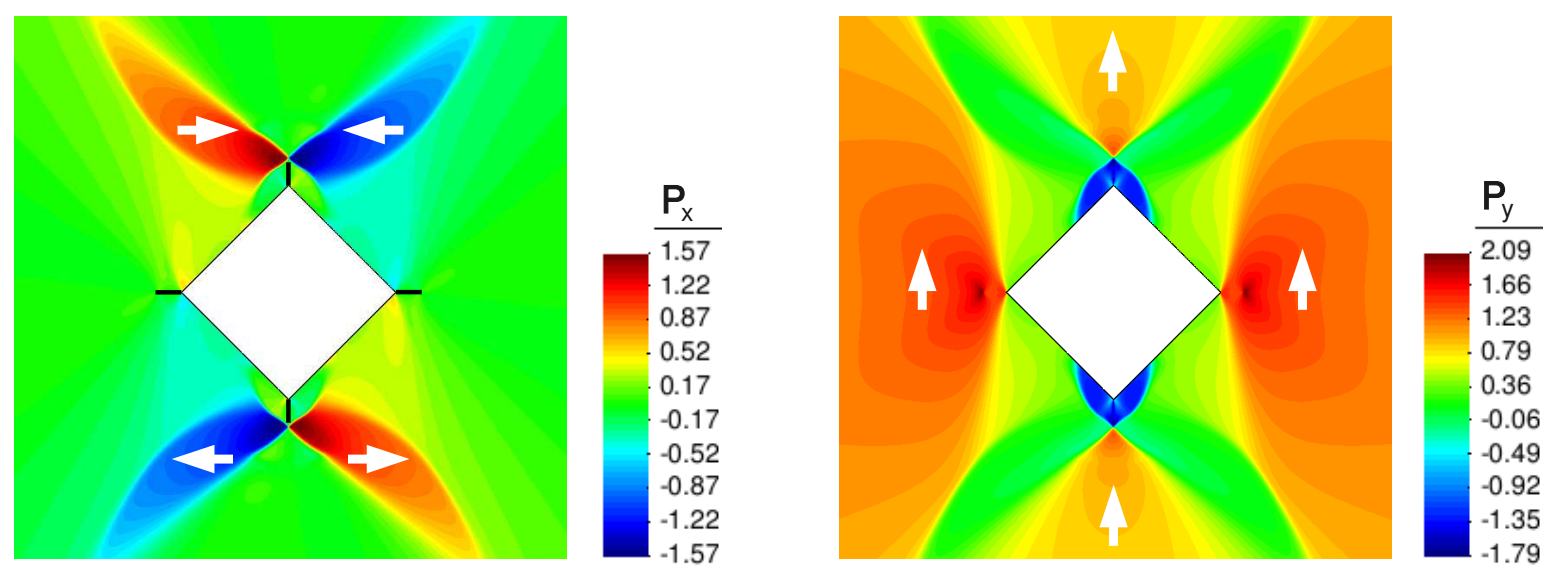

(b)

Figure 6. Two snapshots of the microstructure evolution in an area near the indentation at load steps (a) $w=1.2$ and (b) $w=1.8$. The left and right columns show the horizontal and vertical components of the polarization, respectively. The black lines in the left column indicate the position of the cracks $(v=0)$. The domain orientations are indicated with white arrows.

\section{Appendix A. Weak form of the governing equations}

The weak forms of the gradient flows in (9) and (10), together with the equations for mechanical and electrostatic equilibria in (11) and (12), follow from

$\alpha \int_{\Omega} \dot{p}_{i} \delta p_{i} \mathrm{~d} \Omega=-\delta H[\boldsymbol{u}, \boldsymbol{p}, v, \phi ; \delta \boldsymbol{p}]$ 


$$
\begin{aligned}
& =-\int_{\Omega}\left[\left(v^{2}+\eta\right)\left(\frac{\partial U}{\partial p_{i, j}} \delta p_{i, j}+\frac{\partial W}{\partial p_{i}} \delta p_{i}\right)+\frac{\partial \chi}{\partial p_{i}} \delta p_{i}+\phi, i \delta p_{i}\right] \mathrm{d} \Omega, \\
\beta \int_{\Omega} \dot{v} \delta v \mathrm{~d} \Omega & =-\delta H[\boldsymbol{u}, \boldsymbol{p}, v, \phi ; \delta v] \\
& =-\int_{\Omega} \frac{\partial\left(W_{e}+W_{f}\right)}{\partial v} \delta v \mathrm{~d} \Omega-2 G_{c} \int_{\Omega}\left(\frac{v-1}{4 \kappa} \delta v+\kappa v_{, i} \delta v_{, i}\right) \mathrm{d} \Omega, \\
0 & =\delta H[\boldsymbol{u}, \boldsymbol{p}, v, \phi ; \delta \boldsymbol{u}]=\int_{\Omega} \frac{\partial\left(W_{e}+W_{f}\right)}{\partial \varepsilon_{i j}} \delta \varepsilon_{i j} \mathrm{~d} \Omega, \\
0 & =-\delta H[\boldsymbol{u}, \boldsymbol{p}, v, \phi ; \delta \phi]=\int_{\Omega} \frac{\partial W_{f}}{\partial E_{i}} \delta E_{i} \mathrm{~d} \Omega,
\end{aligned}
$$

where the elastic strain $\varepsilon_{i j}$ and electric field $E_{i}$ are associated with the mechanical displacement $u_{i}$ and the electric potential $\phi$, respectively, as $\varepsilon_{i j}=1 / 2\left(u_{i, j}+u_{j, i}\right)$ and $E_{i}=-\phi_{, i}$.

\section{Acknowledgments}

The authors gratefully acknowledge the support of the Ministerio de Ciencia e Innovación (DPI2010-19145).

\section{References}

[1] Han X L, Li X and Mao S X 2002 Metall. Mater. Trans. A 33 2835-45

[2] Rajapakse R K N D and Zeng X 2001 Acta Mater. 49 877-85

[3] Beom H G and Atluri S N 2003 J. Mech. Phys. Solids 51 1107-25

[4] Sheng J S and Landis C M 2007 Int. J. Fract. 143 161-75

[5] Sun C T and Park S B 2000 Ferroelectrics 248 79-95

[6] Pisarenko G G, Chushko V M and Kovalev S P 1985 J. Am. Ceram. Soc. 68 259-65

[7] Tobin A G and Pak Y E 1993 Proc. SPIE, Smart Struct. Mater 1916 78-86

[8] Wang H Y and Singh R N 1997 J. Appl. Phys. 81 7471-9

[9] Lynch C S 1998 Acta Mater. 46 599-608

[10] Schneider G A and Heyer V 1999 J. Eur. Ceram. Soc. 19 1299-1306

[11] Zhang T Y and Gao C F 2004 Theor. Appl. Fract. Mech. 41 339-79

[12] Schneider G A 2007 Annu. Rev. Mater. Res. 37 491-538

[13] Kuna M 2010 Eng. Fract. Mech. 77 309-26

[14] McMeeking R M and Landis C M 2002 Int. J. Eng. Sci. 40 1553-77

[15] Wang J and Landis C M 2006 J. Mech. Mater. Struct. 1 1075-95 
[16] Hwang S C, Lynch C S and McMeeking R M 1995 Acta Metal. Mater. 43 2073-84

[17] Zhu T and Yang W 1997 Acta Mater. 45 4695-4702

[18] Yang W and Zhu T 1998 J. Mech. Phys. Solids 46 291-311

[19] Zhu T and Yang W 1999 J. Mech. Phys. Solids 47 81-97

[20] Zhang W and Bhattacharya K 2005 Acta Mater. 53 185-98

[21] Schrade D, Mueller R, Xu B and Gross D 2007 Comput. Methods. Appl. Mech. Eng. $1964365-74$

[22] Xu B X, Schrade D, Mueller R and Gross D 2009 Comput. Mater. Sci. 45 832-36

[23] Su Y and Landis C M 2007 J. Mech. Phys. Solids 55 280-305

[24] Dayal K and Bhattacharya K 2007 Acta Mater. 55 1907-17

[25] DeSimone A 1994 J. Intell. Mater. Syst. Struct. 5 787-94

[26] DeSimone A 1996 Z. Angew. Math. Mech. 76 397-400

[27] Song Y C, Soh A K and Ni Y 2007 J. Phys. D: Appl. Phys. 40 1175-82

[28] Wang J and Zhang T Y 2007 Acta Mater. 55 2465-77

[29] Li W and Landis C M 2011 Eng. Fract. Mech. DOI: 10.1016/j.engfracmech.2011.01.002

[30] Arias I, Serebrinsky S and Ortiz M 2006 Acta Mater. 54 975-84

[31] Gao H J, Zhang T Y and Tong P 1997 J. Mech. Phys. Solids 45 491-510

[32] Abdollahi A and Arias I 2011 Acta Mater. DOI: 10.1016/j.actamat.2011.03.030

[33] Francfort G A and Marigo J J 1998 J. Mech. Phys. Solids 46 1319-42

[34] Bourdin B, Francfort G A and Marigo J J 2000 J. Mech. Phys. Solids 48 797-826

[35] Bourdin B 2007 Interfaces Free Bound. 9 411-30

[36] Bourdin B, Francfort G A and Marigo J J 2008 J. Elast. 91 5-148

[37] Xu B X, Schrade D, Gross D and Mueller R 2010 Int. J. Fract. 166 163-72

[38] Amor H, Marigo J J and Maurini C 2009 J. Mech. Phys. Solids 57 1209-29

[39] Abdollahi A and Arias I 2011 In preparation.

[40] Griffith A A 1921 Philos. Trans. Royal Soc. London A221 163-98

[41] Landis C M 2004 Int. J. Solids Struct. 41 6291-315

[42] Vendik O G and Zubko S P 2000 J. Appl. Phys. 88 5343-50

[43] Devonshire A F 1949 Philos. Mag. 40 1040-63

[44] Devonshire A F 1951 Philos. Mag. 42 1065-79

[45] Li Y L, Cross L E and Chen L Q 2005 J. Appl. Phys. 98 064101(1-4) 
[46] Wang Y L, Tagantsev A K, Damjanovic D, Setter N, Yarmarkin V K, Sokolov A I and Lukyanchuk I A 2007 J. Appl. Phys. 101 104115(1-9)

[47] Meschke F, Raddatz O, Kolleck A and Schneider G A 2000 J. Am. Ceram. Soc. 83 $353-61$

[48] Dadvand P, Rossi R and Onate E 2010 Arch. Comput. Methods Eng. 17 253-97

[49] Meschke F, Kolleck A and Schneider G A 1997 J. Eur. Ceram. Soc. 17 1143-9

[50] Fang D N, Jiang Y J, Li S and Sun C T 2007 Acta Mater. 55 5758-67 